\title{
Detection of Extended Spectrum Beta-Lactamases Resistance Genes among Bacteria Isolated from Selected Drinking Water Distribution Channels in Southwestern Nigeria
}

\author{
Ayodele T. Adesoji ${ }^{1}$ and Adeniyi A. Ogunjobi ${ }^{2}$ \\ ${ }^{1}$ Department of Biological Sciences, Federal University Dutsin-Ma, Katsina Road, PMB 5001, Dutsin-Ma, Katsina State, Nigeria \\ ${ }^{2}$ Department of Microbiology, University of Ibadan, Oyo State, Nigeria
}

Correspondence should be addressed to Ayodele T. Adesoji; timmyayus2002@yahoo.com

Received 11 April 2016; Accepted 4 July 2016

Academic Editor: Carla R. Arciola

Copyright (C) 2016 A. T. Adesoji and A. A. Ogunjobi. This is an open access article distributed under the Creative Commons Attribution License, which permits unrestricted use, distribution, and reproduction in any medium, provided the original work is properly cited.

\begin{abstract}
Extended Spectrum Beta-Lactamases (ESBL) provide high level resistance to beta-lactam antibiotics among bacteria. In this study, previously described multidrug resistant bacteria from raw, treated, and municipal taps of DWDS from selected dams in southwestern Nigeria were assessed for the presence of ESBL resistance genes which include $b l a_{\mathrm{TEM}}, b l a_{\mathrm{SHV}}$, and $b l a_{\mathrm{CTX}}$ by PCR amplification. A total of 164 bacteria spread across treated (33), raw (66), and municipal taps (68), belonging to $\alpha$-Proteobacteria, $\beta$-Proteobacteria, $\gamma$-Proteobacteria, Flavobacteriia, Bacilli, and Actinobacteria group, were selected for this study. Among these bacteria, the most commonly observed resistance was for ampicillin and amoxicillin/clavulanic acid (61 isolates). Sixty-one isolates carried at least one of the targeted ESBL genes with $b l a_{\mathrm{TEM}}$ being the most abundant (50/61) and $b l a_{\mathrm{CTX}}$ being detected least (3/61). Klebsiella was the most frequently identified genus (18.03\%) to harbour ESBL gene followed by Proteus (14.75\%). Moreover, combinations of two ESBL genes, $b l a_{\mathrm{SHV}}+b l a_{\mathrm{TEM}}$ or $b l a_{\mathrm{CTX}}+b l a_{\mathrm{TEM}}$, were observed in 11 and 1 isolate, respectively. In conclusion, classic bla $_{\mathrm{TEM}}$ ESBL gene was present in multiple bacterial strains that were isolated from DWDS sources in Nigeria. These environments may serve as foci exchange of genetic traits in a diversity of Gram-negative bacteria.
\end{abstract}

\section{Introduction}

Access to safe drinking water is essential for human health [1]. While access to safe and affordable water should be available to everyone, this remains a challenge in low- and middleincome countries including Nigeria, which is the most populous country in Africa. Safe drinking water is mostly viewed in terms of organic and inorganic contaminants, but also in terms of biological contamination. In this respect, less attention has been given to the role that water may play in the dissemination of antibiotic resistance traits in populations that are exposed to substandard water on a daily basis [2-5].

Arguably, the most clinically important antibiotic resistance genes are those that encode enzymes that hydrolyze $\beta$ lactams (bla genes) [6]. These traits confer high level resistance to $\beta$-lactam antibiotics, which are the most widely used antibiotics in clinical and veterinary practice $[7,8]$. Extended Spectrum $\beta$-Lactamase (ESBL) is group of enzymes that can hydrolyze a variety of $\beta$-lactams including cephalosporins like ceftazidime, cefotaxime, and ceftriaxone and monobactams like aztreonam in addition to penicillin but does not hydrolyze cephamycins like cefoxitin. Most of the ESBL also have the ability to hydrolyze fourth-generation cephalosporins including cefepime [9].

A variety of transferable genes encoding $\beta$-lactamase activity have been described in clinical environments including $b l a_{\mathrm{CTX}-\mathrm{M}}, b l a_{\mathrm{GES}}, b l a_{\mathrm{HER}}, b l a_{\mathrm{OXA}}, b l a_{\mathrm{OXY}}, b l a_{\mathrm{SED}}, b l a_{\mathrm{SHV}}$, $b l a_{\mathrm{SPM}}, b l a_{\mathrm{VEB}}, b l a_{\mathrm{VIM}}$, and $a m p C$ [10]. Among the most common bla genes is the $b l a_{\mathrm{TEM}-1}$ gene, the first described bla gene and a representative of the $b l a_{\text {TEM }}$ group that now consists of more than 220 different distinct variants ("alleles"), which encode different amino acid polymorphisms that extend their 
substrate range (http://www.lahey.org/Studies/temtable.asp) [10].

Previous reports indicated that multidrug resistant bacteria are present in drinking water distribution systems from southwestern Nigeria [3-5]. These bacteria encoded resistance to a diversity of beta-lactams including ceftiofur, ampicillin, and combination of amoxicillin and amoxicillin/ clavulanic acid.

The aim of this study was to genotype MDR bacteria isolated from our previous studies [3-5] for the presence of selected beta-lactamase resistance genes using PCR.

\section{Materials and Methods}

2.1. Dam Description, Sampling, Selection, Isolation, Storage, and Molecular Characterization of Bacteria. The description of sampled dams in this study is in our previous publications [3-5]. Moreover, for clarity of this paper, ninety-six water samples were purposively collected aseptically into sterile screw cap bottles from six selected water distribution systems of dams in Ife, Ede, Asejire, Eleyele, Owena Ondo, and Owena-Idanre in southwestern Nigeria. Samples were collected four times between December 2010 and July 2011 from raw, treated, and two randomly selected municipal distribution taps. Afterwards, samples were serially diluted and plated on Nutrient agar, eosin methylene blue agar (EMB), and Deoxycholate agar (DCA). Thereafter, bacteria were picked with the aim of maximizing the diversity of colony morphology represented from each sample. Picked colonies were restreaked on Nutrient agar to obtain pure cultures. These were subsequently transferred to Nutrient agar slants and also stored in phosphate buffer glycerol at $-80^{\circ} \mathrm{C}$ [3-5]. Molecular characterization of bacteria using $16 \mathrm{~S}$ rDNA sequencing was determined as described in Adesoji et al. [11].

2.2. Antibiotic Susceptibility Testing. Agar dilution assays (also called breakpoint assays) were conducted using LuriaBertani agar with seeded antibiotics used to assess antibiotic susceptibility. Antibiotics concentrations used for Gramnegative bacteria included florfenicol $(16 \mu \mathrm{g} / \mathrm{mL})$, tetracycline $(16 \mu \mathrm{g} / \mathrm{mL})$, streptomycin $(16 \mu \mathrm{g} / \mathrm{mL})$, gentamycin $(16 \mu \mathrm{g} / \mathrm{mL})$, kanamycin $(64 \mu \mathrm{g} / \mathrm{mL})$, chloramphenicol $(32 \mu \mathrm{g} / \mathrm{mL})$, nalidixic acid $(30 \mu \mathrm{g} / \mathrm{mL})$, amoxicillin/clavulanic acid $(32 / 16 \mu \mathrm{g} / \mathrm{mL})$, ceftiofur $(12 \mu \mathrm{g} / \mathrm{mL})$, sulfamethoxazole $(512 \mu \mathrm{g} / \mathrm{mL})$, and sulfamethoxazole/trimethoprim $(76 / 4 \mu \mathrm{g} /$ $\mathrm{mL})$. Antibiotics concentrations used for Gram-positive bacteria include sulfamethoxazole $(512 \mu \mathrm{g} / \mathrm{mL})$, ampicillin $(0.5 \mu \mathrm{g} / \mathrm{mL})$, tetracycline $(16 \mu \mathrm{g} / \mathrm{mL})$, sulfamethoxazole/trimethoprim $(76 / 4 \mu \mathrm{g} / \mathrm{mL})$, gentamycin $(16 \mu \mathrm{g} / \mathrm{mL})$, erythromycin $(8 \mu \mathrm{g} / \mathrm{mL})$, rifampin $(4 \mu \mathrm{g} / \mathrm{mL})$, lincomycin $(4 \mu \mathrm{g} / \mathrm{mL})$, and ciprofloxacin $(4 \mu \mathrm{g} / \mathrm{mL})$. Negative and positive controls used were E. coli strain $\mathrm{K} 12$ and $E$. coli strain $\mathrm{H} 4 \mathrm{H}$, respectively, as we described in our previous studies [3-5, 11].

2.3. Resistance Genotyping. PCR testing was conducted for bacteria having resistance to $\geq 3$ classes of antibiotics including resistance to amoxicillin/clavulanic acid, ceftiofur, or ampicillin. Thereafter, forward and reverse primer specific for selected ESBL genes included $b l a_{\mathrm{SHV}}$ (SHV_F, $5^{\prime}$-GCGAAAGCCAGCTGTCGGGC- $3^{\prime}$ and SHV_R, $5^{\prime}$-GATTGGCGGCGCTGTTATCGC-3), bla $a_{\text {CTX_m }}$ (CTX_F, 5'-GTGCAGTACCAGTAAAGTTATGG-3' $3^{\prime}$ and CTX_R, $5^{\prime}$-CGCAATATCATTGGTGGTGCC- $3^{\prime}$ ), and $b l a_{\text {TEM }}$ (TEM_F, $5^{\prime}$-AAAGATGCTGAAGATCA- $3^{\prime}$ and TEM_R, $5^{\prime}$-TTTGGTATGGCTTCATTC- $3^{\prime}$ ) [12]. Condition for $b l a_{\mathrm{SHV}}$ PCR included $1 \mathrm{~min}$ denaturation $\left(95^{\circ} \mathrm{C}\right.$ followed by 30 cycles of $96^{\circ} \mathrm{C}$ for $30 \mathrm{~s}, 62^{\circ} \mathrm{C}$ for $30 \mathrm{~s}$, and $72^{\circ} \mathrm{C}$ for $30 \mathrm{~s}$ and final extension of $72^{\circ} \mathrm{C}$ for $10 \mathrm{~min}$. Conditions were identical for other assays except the annealing temperatures which were $55^{\circ} \mathrm{C}$ and $44^{\circ} \mathrm{C}$ for $b l a_{\mathrm{CTX}-\mathrm{M}}$ and $b l a_{\mathrm{TEM}}$, respectively. Afterwards, PCR products were separated, sized, and visualized by using $1 \%$ agarose gel electrophoresis to confirm amplification.

\section{Results}

3.1. Bacteria Isolates. Isolates used in this study were selected from our previous studies [3-5] and represented $\alpha$-Proteobacteria, $\beta$-Proteobacteria, $\quad \gamma$-Proteobacteria, Flavobacteriia, Bacilli, and Actinobacteria with 33, 66, and 68 being isolated from all treated, raw, and municipal taps, respectively (Table 1). Proteus was the most frequent $(18.18 \%)$ isolated Gram-negative genus from the treated water while Klebsiella was the most frequently $(15.15 \%)$ isolated genus from raw water. Bacillus was the most common isolated Gram-positive genus for treated and municipal water.

3.2. PCR-Positive Isolates. In this study, 61 isolates out of 164 MDR isolates were PCR-positive for at least one targeted gene. Highest occurrence of bla gene among Gramnegative bacteria compared to Gram-positive bacteria was observed. Most commonly isolated genus carrying bla gene is Klebsiella (18.03\%) followed by Proteus spp. (14.75\%). $B l a_{\text {TEM }}$ was detected in the majority of beta-lactam resistant isolates $(50 / 61)$ while $b l a_{\text {CTX }}$ was rarely detected $(3 / 61)$ (Table 2). A combination of two genes, $b l a_{\mathrm{SHV}}+b l a_{\mathrm{TEM}}$ or $b l a_{\mathrm{CTX}}+b l a_{\mathrm{TEM}}$, was observed in 11 and 1 bacteria, respectively (Table 2). Other genera, including Aquitalea, Comamonas, Enterobacter, Leucobacter, Lysinibacillus, Pantoea, Pseudochrobactrum, Sphingobacterium, and Ralstonia, were tested but were PCR-negative for resistance genes.

\section{Discussion}

The hazard associated with the pathogenicity of microbes is aggravated by its ability to resist destruction by antibiotics [2]. In this study, beta-lactamase producing bacteria and genes (i.e., $b l a_{\mathrm{CTX}}$ and $b l a_{\mathrm{TEM}}$ ) were detected from every sampled water distribution system. This is similar to the report of $\mathrm{Xi}$ et al. [13] who also investigated the prevalence and dynamics of heterotrophic antibiotics resistance bacteria and genes in drinking water source and treated drinking water using culture-dependent methods and molecular techniques. The authors observed the presence of $b l a_{\mathrm{TEM}}$ and $b l a_{\mathrm{SHV}}$ genes in all water samples except one, which is evidence that these genes are distributed widely in drinking water systems. This is similar to what we also reported in Table 3, showing the spread of these beta-lactamase resistance genes among all 
TABLE 1: Classes and families of selected bacteria.

\begin{tabular}{|c|c|c|c|}
\hline Source & Class & Family & Number (\% of total from source) \\
\hline \multirow{13}{*}{ Raw water } & $\alpha$-Proteobacteria & Brucellaceae & $1(1.51)$ \\
\hline & \multirow{2}{*}{$\beta$-Proteobacteria } & Alcaligenaceae & $9(13.64)$ \\
\hline & & Neisseriaceae & $1(1.51)$ \\
\hline & \multirow{4}{*}{$\gamma$-Proteobacteria } & Enterobacteriaceae & $27(40.91)$ \\
\hline & & Moraxellaceae & $2(3.03)$ \\
\hline & & Aeromonadaceae & $6(9.09)$ \\
\hline & & Xanthomonadaceae & $2(3.03)$ \\
\hline & Flavobacteriia & Myroidaceae & $1(1.51)$ \\
\hline & Uncultured bacteria clone & & $3(4.55)$ \\
\hline & \multirow{2}{*}{ Bacilli } & Bacillaceae & $10(15.15)$ \\
\hline & & Staphylococcaceae & $3(4.55)$ \\
\hline & Actinobacteria & Microbacteriaceae & $1(1.51)$ \\
\hline & Total raw water & & 66 \\
\hline \multirow{9}{*}{ Treated water } & $\alpha$-Proteobacteria & Caulobacteraceae & $1(3.03)$ \\
\hline & \multirow{2}{*}{$\beta$-Proteobacteria } & Alcaligenaceae & $5(15.15)$ \\
\hline & & Neisseriaceae & $1(3.03)$ \\
\hline & $\gamma$-Proteobacteria & Enterobacteriaceae & $10(30.30)$ \\
\hline & Flavobacteriia & Myroidaceae & $1(3.03)$ \\
\hline & Uncultured bacteria clone & & $2(6.06)$ \\
\hline & \multirow{2}{*}{ Bacilli } & Bacillaceae & $12(36.36)$ \\
\hline & & Staphylococcaceae & $1(3.03)$ \\
\hline & Total treated water & & 33 \\
\hline \multirow{10}{*}{ Municipal taps } & $\alpha$-Proteobacteria & Caulobacteraceae & $1(1.47)$ \\
\hline & \multirow{2}{*}{$\beta$-Proteobacteria } & Alcaligenaceae & $7(10.29)$ \\
\hline & & Neisseriaceae & $3(4.41)$ \\
\hline & \multirow{2}{*}{$\gamma$-Proteobacteria } & Enterobacteriaceae & $21(30.88)$ \\
\hline & & Moraxellaceae & $6(8.82)$ \\
\hline & Flavobacteriia & Myroidaceae & $3(4.41)$ \\
\hline & \multicolumn{3}{|l|}{ Uncultured bacteria clone } \\
\hline & \multirow{2}{*}{ Bacilli } & Bacillaceae & $26(38.24)$ \\
\hline & & Staphylococcaceae & $1(1.47)$ \\
\hline & Total municipal tap & & 68 \\
\hline
\end{tabular}

Note: identification was based on $16 \mathrm{~S}$ rDNA sequencing. These bacteria were obtained from our previous works [3-5].

raw, final, and municipal tap sources. The results showed that even among bacteria from the municipal tap and final treated water from the dam which are the point of consumer consumption $b l a_{\mathrm{TEM}}$ and $b l a_{\mathrm{CTX}}$ occurred among bacteria from these sources in high number. Xi et al. [13] also observed selective increases in the levels of both genes in tap water due to either water treatment or regrowth within drinking water distribution systems. This, as they therefore reported, suggested the spread of at least some beta-lactam-resistant determinants through drinking water distribution systems. However, in this study, it is important to point out that every site is different for numerous variables making it impossible to derive meaningful correlations between water treatment practices and the occurrence of beta-lactamase resistance genes. Given these differences, the only practical means to assess the effects of water treatment practices (which is not our goal with this paper) would be to test changes with experimental manipulation. We have been reluctant to provide significant detail of the sample sites precisely because we do not wish to imply that there are defendable correlations between occurrence and site characteristics, nor do we wish to encourage readers to draw such inferences.

Moreover, we observed that there were beta-lactam resistant strains that were negative for the PCR assays used in this study. The most commonly detected bla genes were $b l a_{\text {TEM }}$ and $b l a_{\mathrm{SHV}}$ among Klebsiella. This finding is contrary to previous reports [14-16]. They observed dominance of $b l a_{\mathrm{CTX}}$ among non-TEM and SHV bacteria from clinical environment which were similar to what Ojdana et al. [17] reported among clinical samples from Poland. Additionally, studies on Pseudomonas spp. isolated from these water distribution systems also observed a higher occurrence of $b l a_{\text {TEM }}(40.9 \%)$ and $b l a_{\text {СтХ }}(27.3 \%)$ while none of the pseudomonads showed the presence of $b l a_{\text {Стх }}$ [18]. Our observation of the highest occurrence of bla gene among Gram-negative bacteria, when compared to Gram-positive bacteria, in this study is similar 
TABLE 2: Characterization of a number of cultured bacterial isolates encoding different ESBL genotypes.

\begin{tabular}{|c|c|c|c|c|c|}
\hline Genus/species/accession number & Source & Resistant phenotypes & $b l a_{\mathrm{TEM}}$ & $b l a_{\mathrm{SHV}}$ & $b l a_{\mathrm{CTX}}$ \\
\hline \multicolumn{6}{|c|}{$\operatorname{Dam} 1^{\mathrm{a}}$} \\
\hline Escherichia coli AP010960.1 & $\begin{array}{l}\text { DAM } 1 \\
\text { IRW }\end{array}$ & T, AM, S, C, N, SXT, SU & $b l a_{\mathrm{TEM}}$ & & \\
\hline Uncultured bacterium clone JN595783.1 & $\begin{array}{l}\text { DAM } 1 \\
\text { IFFW }\end{array}$ & T, FF, AM, G, SU & $b l a_{\mathrm{TEM}}$ & & \\
\hline Bacillus thuringiensis JN377782.1 & $\begin{array}{l}\text { DAM } 1 \\
\text { IFFW }\end{array}$ & $\begin{array}{l}\text { SU, AM, T, E, SXT, RIF, LIN, } \\
\text { GEN }\end{array}$ & $b l a_{\mathrm{TEM}}$ & & \\
\hline Brevundimonas diminuta EU545397.1 & $\begin{array}{l}\text { DAM } 1 \\
\text { IFFW }\end{array}$ & S, G, K, N, AM, SXT, SU & & $b l a_{\mathrm{SHV}}$ & \\
\hline Proteus mirabilis AB626123.1 & $\begin{array}{l}\text { DAM } 1 \\
\text { IFFW }\end{array}$ & $\begin{array}{c}\mathrm{FF}, \mathrm{T}, \mathrm{S}, \mathrm{G}, \mathrm{K}, \mathrm{C}, \mathrm{AMC}, \mathrm{AM}, \mathrm{SU} \\
\mathrm{SXT}\end{array}$ & $b l a_{\mathrm{TEM}}$ & & \\
\hline Bacillus thuringiensis JN377782.1 & $\begin{array}{l}\text { DAM } 1 \\
\text { IFM1 }\end{array}$ & SU, AM, E, SXT, RIF, LIN & $b l a_{\mathrm{TEM}}$ & $b l a_{\mathrm{SHV}}$ & \\
\hline \multicolumn{6}{|c|}{$\operatorname{Dam} 2^{\mathrm{b}}$} \\
\hline Bacillus altitudinis HQ432811.1 & $\begin{array}{l}\text { DAM } 2 \\
\text { EDRW }\end{array}$ & SU, E, RIF, LIN, AM & & $b l a_{\mathrm{SHV}}$ & \\
\hline Bordetella sp. HQ840720.1 & $\begin{array}{l}\text { DAM } 2 \\
\text { EDRW }\end{array}$ & T, FF, S, C, N, CEF, AM, SXT, SU & $b l a_{\mathrm{TEM}}$ & & \\
\hline Proteus vulgaris JN630888.1 & $\begin{array}{l}\text { DAM } 2 \\
\text { EDRW }\end{array}$ & T, AM, SXT, SU & $b l a_{\mathrm{TEM}}$ & & \\
\hline Staphylococcus sp. JN695710.1 & $\begin{array}{l}\text { DAM } 2 \\
\text { EDRW }\end{array}$ & SU, T, E, SXT, RIF, LIN, AM & $b l a_{\mathrm{TEM}}$ & & \\
\hline Stenotrophomonas maltophilia JN703732.1 & $\begin{array}{l}\text { DAM } 2 \\
\text { EDRW }\end{array}$ & T, S, K, CEF, AM, AMC, SU & $b l a_{\mathrm{TEM}}$ & $b l a_{\mathrm{SHV}}$ & \\
\hline Bacillus cereus AP007209.1 & $\begin{array}{l}\text { DAM } 2 \\
\text { EDFW }\end{array}$ & SU, AM, T, E, SXT, RIF, LIN & $b l a_{\mathrm{TEM}}$ & & \\
\hline Morganella sp. GQ179706.1 & $\begin{array}{l}\text { DAM } 2 \\
\text { EDFW }\end{array}$ & T, S, AM, SXT, SU & $b l a_{\mathrm{TEM}}$ & & \\
\hline Psychrobacter sp. HQ730697.1 & $\begin{array}{l}\text { DAM } 2 \\
\text { EDM2 }\end{array}$ & T, S, CEF, AM, SXT, SU & $b l a_{\mathrm{TEM}}$ & & \\
\hline \multicolumn{6}{|c|}{$\operatorname{Dam} 3^{\mathrm{c}}$} \\
\hline Alcaligenes faecalis JN162124.1 & $\begin{array}{l}\text { DAM } 3 \\
\text { ARW }\end{array}$ & S, CEF, AM, SXT, SU & & $b l a_{\mathrm{SHV}}$ & \\
\hline Klebsiella pneumoniae AB675600.1 & $\begin{array}{l}\text { DAM } 3 \\
\text { ARW }\end{array}$ & $\begin{array}{c}\text { FF, T, S, C, AMC, CEF, AM, SU, } \\
\text { SXT }\end{array}$ & $b l a_{\mathrm{TEM}}$ & & \\
\hline Leucobacter komagatae AJ746337.1 & $\begin{array}{l}\text { DAM } 3 \\
\text { ARW }\end{array}$ & T, S, AM, G, K, SXT, N, SU & $b l a_{\mathrm{TEM}}$ & & \\
\hline Proteus mirabilis AB626123.1 & $\begin{array}{l}\text { DAM } 3 \\
\text { ARW }\end{array}$ & $\mathrm{T}, \mathrm{S}, \mathrm{AM}, \mathrm{N}, \mathrm{SXT}, \mathrm{SU}$ & $b l a_{\mathrm{TEM}}$ & & \\
\hline Uncultured bacterium clone JN595783.1 & $\begin{array}{l}\text { DAM } 3 \\
\text { ARW }\end{array}$ & $\begin{array}{c}\text { T, G, K, C, N, CEF, AM, SXT, } \\
\text { AMC, SU }\end{array}$ & $b l a_{\mathrm{TEM}}$ & & \\
\hline Bacillus pumilus EF010673.1 & $\begin{array}{l}\text { DAM } 3 \\
\text { AFW }\end{array}$ & SU, AM, T, E, SXT, RIF, LIN & $b l a_{\mathrm{TEM}}$ & & \\
\hline Klebsiella pneumoniae JF919909.1 & $\begin{array}{l}\text { DAM } 3 \\
\text { AFW }\end{array}$ & T, S, C, AM, SXT, SU & & $b l a_{\mathrm{SHV}}$ & \\
\hline Myroides odoratus AB517709.1 & $\begin{array}{l}\text { DAM } 3 \\
\text { AFW }\end{array}$ & $\begin{array}{c}\mathrm{FF}, \mathrm{T}, \mathrm{S}, \mathrm{G}, \mathrm{K}, \mathrm{C}, \mathrm{AM}, \mathrm{SXT}, \mathrm{AMC} \text {, } \\
\text { SU }\end{array}$ & $b l a_{\mathrm{TEM}}$ & & \\
\hline Proteus vulgaris JN630888.1 & $\begin{array}{l}\text { DAM } 3 \\
\text { AFW }\end{array}$ & $\begin{array}{c}\text { FF, T, S, C, N, CEF, AM, SXT, } \\
\text { AMC, SU }\end{array}$ & $b l a_{\mathrm{TEM}}$ & & \\
\hline Acinetobacter calcoaceticus & $\begin{array}{l}\text { DAM } 3 \\
\text { AM2 }\end{array}$ & S, AMC, AM, SU & $b l a_{\mathrm{TEM}}$ & $b l a_{\mathrm{SHV}}$ & \\
\hline Chromobacterium sp. AB426118.1 & $\begin{array}{l}\text { DAM } 3 \\
\text { AM1 }\end{array}$ & $\begin{array}{c}\text { T, S, CEF, AM, SXT, SU, AMC, } \\
\text { SU }\end{array}$ & $b l a_{\mathrm{TEM}}$ & & \\
\hline Klebsiella pneumoniae JF513171.1 & $\begin{array}{l}\text { DAM } 3 \\
\text { AM2 }\end{array}$ & $\begin{array}{l}\text { FF, C, CEF, AM, SXT, AMC, SU, } \\
\text { AMC, SU }\end{array}$ & $b l a_{\mathrm{TEM}}$ & $b l a_{\mathrm{SHV}}$ & \\
\hline
\end{tabular}


TABle 2: Continued.

\begin{tabular}{|c|c|c|c|c|c|}
\hline Genus/species/accession number & Source & Resistant phenotypes & $b l a_{\mathrm{TEM}}$ & $b l a_{\mathrm{SHV}}$ & $b l a_{\mathrm{CTX}}$ \\
\hline \multicolumn{6}{|c|}{$\operatorname{Dam} 4^{\mathrm{d}}$} \\
\hline Aeromonas caviae AB626132.1 & $\begin{array}{l}\text { DAM } 4 \\
\text { ERW }\end{array}$ & T, S, AM, SXT, N, AMC, SU & $b l a_{\mathrm{TEM}}$ & & \\
\hline Alcaligenes faecalis HQ161777.1 & $\begin{array}{c}\text { DAM } 4 \\
\text { ERW }\end{array}$ & $\mathrm{T}, \mathrm{S}, \mathrm{K}, \mathrm{AM}, \mathrm{SU}$ & $b l a_{\mathrm{TEM}}$ & & \\
\hline Alcaligenes faecalis JN162124.1 & $\begin{array}{c}\text { DAM } 4 \\
\text { ERW }\end{array}$ & $\mathrm{T}, \mathrm{S}, \mathrm{K}, \mathrm{AM}, \mathrm{SU}$ & $b l a_{\mathrm{TEM}}$ & & \\
\hline Klebsiella pneumoniae JN545039.1 & $\begin{array}{c}\text { DAM } 4 \\
\text { ERW }\end{array}$ & S, CEF, AM, SXT, AMC, SU & $b l a_{\mathrm{TEM}}$ & & \\
\hline Klebsiella pneumoniae JN545039.1 & $\begin{array}{c}\text { DAM } 4 \\
\text { ERW }\end{array}$ & $\mathrm{T}, \mathrm{K}, \mathrm{N}, \mathrm{AM}, \mathrm{SU}$ & $b l a_{\mathrm{TEM}}$ & $b l a_{\mathrm{SHV}}$ & \\
\hline Klebsiella pneumoniae JF513172.1 & $\begin{array}{l}\text { DAM } 4 \\
\text { ERW }\end{array}$ & T, FF, S, C, AM, SXT, AMC, SU & $b l a_{\mathrm{TEM}}$ & $b l a_{\mathrm{SHV}}$ & \\
\hline Morganella morganii FJ971868.1 & $\begin{array}{l}\text { DAM } 4 \\
\text { ERW }\end{array}$ & T, S, K, CEF, AM, SXT, AMC, SU & $b l a_{\mathrm{TEM}}$ & & \\
\hline Proteus vulgaris JN630888.1 & $\begin{array}{c}\text { DAM } 4 \\
\text { ERW }\end{array}$ & T, C, CEF, AM & $b l a_{\mathrm{TEM}}$ & & \\
\hline Proteus mirabilis GU420988.1 & $\begin{array}{l}\text { DAM } 4 \\
\text { ERW }\end{array}$ & T, S, K, N, AM, SXT, AMC, SU & $b l a_{\mathrm{TEM}}$ & & \\
\hline Proteus vulgaris JN630888.1 & $\begin{array}{l}\text { DAM } 4 \\
\text { ERW }\end{array}$ & $\begin{array}{c}\text { FF, T, S, G, K, C, AM, SXT, N, } \\
\text { AMC, SU }\end{array}$ & $b l a_{\mathrm{TEM}}$ & & \\
\hline Providencia vermicola NR_042415.1 & $\begin{array}{l}\text { DAM } 4 \\
\text { ERW }\end{array}$ & T, G, AM, SU & $b l a_{\mathrm{TEM}}$ & & \\
\hline Trabulsiella guamensis AB273737.1 & $\begin{array}{l}\text { DAM } 4 \\
\text { ERW }\end{array}$ & T, C, CEF, AM, SU, SXT & & $b l a_{\mathrm{SHV}}$ & \\
\hline \multicolumn{6}{|c|}{$\operatorname{Dam} 5^{\mathrm{e}}$} \\
\hline Klebsiella sp. JN036433.1 & $\begin{array}{l}\text { DAM } 5 \\
\text { OWODFW }\end{array}$ & T, FF, S, C, AM, SXT, AMC, SU & $b l a_{\mathrm{TEM}}$ & $b l a_{\mathrm{SHV}}$ & \\
\hline Alcaligenes faecalis JN162124.1 & $\begin{array}{c}\text { DAM } 5 \\
\text { OWODM2 }\end{array}$ & T, S, G, K, C, AM, SXT, SU & $b l a_{\mathrm{TEM}}$ & & \\
\hline Escherichia coli CР003034.1 & $\begin{array}{c}\text { DAM } 5 \\
\text { OWODM2 }\end{array}$ & $\mathrm{T}, \mathrm{AM}, \mathrm{AMC}, \mathrm{SU}$ & $b l a_{\mathrm{TEM}}$ & & \\
\hline Escherichia coli CР003034.1 & $\begin{array}{c}\text { DAM } 5 \\
\text { OWODM2 }\end{array}$ & $\mathrm{T}, \mathrm{AM}, \mathrm{AMC}, \mathrm{SU}$ & $b l a_{\mathrm{TEM}}$ & & \\
\hline Morganella morganii AM931264.1 & $\begin{array}{l}\text { DAM } 5 \\
\text { OWODM1 }\end{array}$ & T, S, CEF, AM, SXT, AMC, SU & $b l a_{\mathrm{TEM}}$ & & \\
\hline Morganella morganii AB089245.1 & $\begin{array}{c}\text { DAM } 5 \\
\text { OWODM3 }\end{array}$ & T, S, K, CEF, SXT, AMC, SU & $b l a_{\mathrm{TEM}}$ & & \\
\hline Myroides odoratus AB517709.1 & $\begin{array}{c}\text { DAM } 5 \\
\text { OWODM3 }\end{array}$ & $\begin{array}{c}\mathrm{T}, \mathrm{S}, \mathrm{G}, \mathrm{K}, \mathrm{CEF}, \mathrm{AM}, \mathrm{SXT}, \mathrm{AMC} \text {, } \\
\text { SU }\end{array}$ & $b l a_{\mathrm{TEM}}$ & & \\
\hline Serratia marcescens FJ607982.1 & $\begin{array}{c}\text { DAM } 5 \\
\text { OWODM3 }\end{array}$ & T, AM, AMC, CEF, SU & & $b l a_{\mathrm{SHV}}$ & \\
\hline \multicolumn{6}{|c|}{$\operatorname{Dam} 6^{\mathrm{f}}$} \\
\hline Acinetobacter baumannii JF919866.1 & $\begin{array}{l}\text { DAM } 6 \\
\text { OWIRW }\end{array}$ & T, FF, S, C, AM, SXT, AMC, SU & $b l a_{\mathrm{TEM}}$ & \multirow{4}{*}{\multicolumn{2}{|c|}{$b l a_{\mathrm{CTX}}$}} \\
\hline Bacillus thuringiensis JN377782.1 & $\begin{array}{l}\text { DAM } 6 \\
\text { OWIRW }\end{array}$ & $\begin{array}{l}\text { SU, AM, T, SXT, RIF, LIN, CIP, } \\
\text { GEN }\end{array}$ & $b l a_{\mathrm{TEM}}$ & & \\
\hline Klebsiella sp. DQ989215.2 & $\begin{array}{l}\text { DAM } 6 \\
\text { OWIRW }\end{array}$ & T, S, AM, SXT, SU & $b l a_{\mathrm{TEM}}$ & & \\
\hline Klebsiella pneumoniae СР002910.1 & $\begin{array}{l}\text { DAM } 6 \\
\text { OWIRW }\end{array}$ & T, S, C, AM, SXT, AMC, SU & $b l a_{\mathrm{TEM}}$ & & \\
\hline
\end{tabular}


TABLE 2: Continued.

\begin{tabular}{|c|c|c|c|c|c|}
\hline Genus/species/accession number & Source & Resistant phenotypes & $b l a_{\mathrm{TEM}}$ & $b l a_{\mathrm{SHV}}$ & $b l a_{\mathrm{CTX}}$ \\
\hline Klebsiella oxytoca JF317350.1 & $\begin{array}{l}\text { DAM } 6 \\
\text { OWIRW }\end{array}$ & K, AM, SXT, SU & & & $b l a_{\mathrm{CTX}}$ \\
\hline Morganella morganii AB089245.1 & $\begin{array}{l}\text { DAM } 6 \\
\text { OWIRW }\end{array}$ & T, S, AM, AMC, SU & & & $b l a_{\text {СТХ }}$ \\
\hline Proteus vulgaris JN630888.1 & $\begin{array}{l}\text { DAM } 6 \\
\text { OWIRW }\end{array}$ & $\begin{array}{c}\text { T, FF, S, C, CEF, SXT, N, AMC, } \\
\text { SU }\end{array}$ & $b l a_{\mathrm{TEM}}$ & & \\
\hline Serratia marcescens JF429936.1 & $\begin{array}{l}\text { DAM } 6 \\
\text { OWIRW }\end{array}$ & T, S, C, CEF, AM, AMC, SU & & $b l a_{\mathrm{SHV}}$ & \\
\hline Uncultured bacterium JN595068.1 & $\begin{array}{l}\text { DAM } 6 \\
\text { OWIRW }\end{array}$ & S, G, K, AM, SU & $b l a_{\mathrm{TEM}}$ & & \\
\hline Bacillus altitudinis HQ432811.1 & $\begin{array}{l}\text { DAM } 6 \\
\text { OWIFW }\end{array}$ & SU, AM, T, E, SXT, RIF, LIN & $b l a_{\mathrm{TEM}}$ & $b l a_{\mathrm{SHV}}$ & \\
\hline Alcaligenes sp. JF707602.1 & $\begin{array}{l}\text { DAM } 6 \\
\text { OWIM1 }\end{array}$ & T, S, G, K, CEF, AM, AMC, SU & $b l a_{\mathrm{TEM}}$ & & \\
\hline Alcaligenes faecalis HM145896.1 & $\begin{array}{l}\text { DAM } 6 \\
\text { OWIM1 }\end{array}$ & T, S, G, K, CEF, AM, AMC, SU & $b l a_{\mathrm{TEM}}$ & $b l a_{\mathrm{SHV}}$ & \\
\hline Alcaligenes sp. JF303893.1 & $\begin{array}{l}\text { DAM } 6 \\
\text { OWIM2 }\end{array}$ & $\mathrm{T}, \mathrm{S}, \mathrm{G}, \mathrm{K}, \mathrm{N}, \mathrm{CEF}, \mathrm{AM}, \mathrm{SU}$ & & $b l a_{\mathrm{SHV}}$ & \\
\hline Citrobacter freundii JN644567.1 & $\begin{array}{l}\text { DAM } 6 \\
\text { OWIM1 }\end{array}$ & T, S, AM, SXT, N, AMC, SU & $b l a_{\mathrm{TEM}}$ & & \\
\hline Klebsiella pneumoniae CР002910.1 & $\begin{array}{l}\text { DAM } 6 \\
\text { OWIM1 }\end{array}$ & T, S, CEF, AM, SU & & $b l a_{\mathrm{SHV}}$ & \\
\hline Proteus mirabilis AB626123.1 & $\begin{array}{c}\text { DAM } 6 \\
\text { OWIM2 }\end{array}$ & T, S, C, N, AMC, SXT & & $b l a_{\mathrm{SHV}}$ & \\
\hline
\end{tabular}

Codes. ${ }^{a}$ Obafemi Awolowo University, Ife, Osun State; IRW = Ife raw water; IFFW = Ife treated water; IFM1 = Ife municipal tap 1; IFM2 = Ife municipal tap 2; ${ }^{\mathrm{b}}$ Ede, Osun State; EDRW = Ede raw water; EDFW = Ede treated water; EDM1 = Ede municipal tap 1; EDM2 = Ede municipal tap 2; ${ }^{\mathrm{c}}$ Asejire, Oyo State, Nigeria; ARW = Asejire raw water; AFW = Asejire treated water; AM1 = Asejire municipal tap 1; AM2 = Asejire municipal tap 2; ${ }^{\mathrm{d}}$ Eleyele, Oyo State; ERW = Eleyele raw water; EFW = Eleyele treated water; EM1 = Eleyele municipal tap 1; EM2 = Eleyele municipal tap 2; ${ }^{\mathrm{e} O w e n a}$ Ondo, Ondo State; OWODRW = Owena Ondo raw water; OWODFW = Owena Ondo treated water; OWODM1 = Owena ondo municipal tap 1; OWODM2 = Owena Ondo municipal tap 2; ${ }^{f}$ Owena-Idanre, Ondo State; OWIRW = Owena-Idanre raw water; OWIFW = Owena-Idanre treated water; OWIM1 = Owena-Idanre municipal tap 1; OWIM2 $=$ Owena-Idanre municipal tap 2. Note: bacteria was identified to the genus level by $16 \mathrm{~S}$ rDNA partial sequence.

Ampicillin (AM); ceftiofur (CEF); chloramphenicol (C); florfenicol (FF); kanamycin (K); streptomycin (S); gentamycin (GEN); tetracycline (T); nalidixic acid (N); sulfamethoxazole (SU); sulfamethoxazole/trimethoprim (SXT); amoxicillin/clavulanic acid (AMC); erythromycin (E); rifampin (RIF); lincomycin (LIN); ciprofloxacin (CIP).

to reports of $[19,20]$. These authors also confirm $b l a_{\mathrm{TEM}}$ that was frequently detected among Gram-negative bacteria from this study as the most common bla gene in their studies.

In this study, environmental bacteria belonging to each of these genera Bordetella, Brevundimonas, Chromobacterium, Providencia, Psychrobacter, Stenotrophomonas, Trabulsiella, and Aeromonas possess at least one of the beta-lactamase resistance genes tested; the most common among them is $b l a_{\mathrm{TEM}}$. Occurrence of this gene in these environmental isolates is contrary to the report that ESBL production is mostly found to occur among enteric species [21]. The first bla genes ( $b l a_{\mathrm{BOR}-1}$ and $\left.b l a_{\mathrm{OXA}-2}\right)$ were reported in Bordetella by Kadlec et al. [22]. However, we did not come across any publication where $b l a_{\mathrm{TEM}}$ has been reported in this bacterium. This could be the first report of this gene in this bacterium. Nevertheless, $b l a_{\mathrm{TEM}}$ has been reported in Providencia [23], Stenotrophomonas [24], and Aeromonas [25]. In fact, another bla gene such as $b l a_{\mathrm{LI}}$ has been reported in Stenotrophomonas from China [26] and $b l a_{\mathrm{SHV}}$ and $b l a_{\mathrm{CTX}-\mathrm{M}}$ have been reported in Aeromonas [25]. Moreover, no bla $a_{\mathrm{SHV}}$ has also been reported in Trabulsiella; this report probably might be its first description.

The association of more than one $\beta$-lactamase within the same isolate has been reported $[27,28]$. However, from our studies the most common of this association is bla $a_{\mathrm{SHV}}+$ $b l a_{\mathrm{TEM}}$. This was detected among Acinetobacter, Alcaligenes, Bacillus, Klebsiella, and Stenotrophomonas while the combination of $b l a_{\mathrm{CTX}}$ and $b l a_{\mathrm{TEM}}$ was only observed in Klebsiella. This occurrence denotes the wider dissemination of these bla genes probably due to involvement of genetic element in mobilization of these genes [29]. These same authors [29] also observed various combinations of $b l a_{\mathrm{CTX}}, b l a_{\mathrm{TEM}}$, and $b l a_{\mathrm{SHV}}$ in Klebsiella from clinical isolates in India.

The occurrence of ESBL genes among bacteria from this study has a public health implication. Previous studies have shown that potential ESBL species such as K. pneumonia, the most frequent bacterium with bla gene in this study, and $E$. coli have a high tendency to possess and transfer bla genes [30]. However, this may occur through conjugation because the genes are often found in mobile elements like 
TABLE 3: Number of bacteria and genes observed from all raw, treated, and municipal taps carrying at least one bla gene tested for in this study.

\begin{tabular}{|c|c|c|c|c|c|c|c|c|c|c|c|c|}
\hline \multirow{2}{*}{ Genus } & \multicolumn{4}{|c|}{ bla genes from raw water } & \multicolumn{4}{|c|}{ bla genes from final water } & \multicolumn{4}{|c|}{ bla genes from municipal taps } \\
\hline & $\begin{array}{l}\text { Bacteria } \\
\text { number }\end{array}$ & $b l a_{\mathrm{TEM}}$ & $b l a_{\mathrm{SHV}}$ & $b l a_{\mathrm{CTX}}$ & $\begin{array}{l}\text { Bacteria } \\
\text { number }\end{array}$ & $b l a_{\mathrm{TEM}}$ & $b l a_{\mathrm{SHV}}$ & $b l a_{\mathrm{CTX}}$ & $\begin{array}{l}\text { Bacteria } \\
\text { number }\end{array}$ & $b l a_{\mathrm{TEM}}$ & $b l a_{\mathrm{SHV}}$ & $b l a_{\mathrm{CTX}}$ \\
\hline Acinetobacter spp. & 1 & 1 & 0 & 0 & 0 & 0 & 0 & 0 & 1 & 1 & 1 & 0 \\
\hline Aeromonas spp. & 1 & 1 & 0 & 0 & 0 & 0 & 0 & 0 & 0 & 0 & 0 & 0 \\
\hline Alcaligenes spp. & 3 & 2 & 1 & 0 & 0 & 0 & 0 & 0 & 4 & 3 & 1 & 0 \\
\hline Bacillus spp. & 2 & 1 & 2 & 0 & 4 & 4 & 1 & 0 & 1 & 1 & 1 & 0 \\
\hline Bordetella spp. & 1 & 1 & 0 & 0 & 0 & 0 & 0 & 0 & 0 & 0 & 0 & 0 \\
\hline Brevundimonas spp. & 0 & 0 & 0 & 0 & 1 & 0 & 1 & 0 & 0 & 0 & 0 & 0 \\
\hline Chromobacterium spp. & 0 & 0 & 0 & 0 & 0 & 0 & 0 & 0 & 1 & 1 & 0 & 0 \\
\hline Citrobacter spp. & 0 & 0 & 0 & 0 & 0 & 0 & 0 & 0 & 1 & 1 & 0 & 0 \\
\hline E. coli & 1 & 1 & 0 & 0 & 0 & 0 & 0 & 0 & 2 & 2 & 0 & 0 \\
\hline Klebsiella spp. & 7 & 6 & 3 & 1 & 2 & 0 & 1 & 0 & 2 & 1 & 2 & 0 \\
\hline Leucobacter spp. & 1 & 1 & 0 & 0 & 0 & 0 & 0 & 0 & 0 & 0 & 0 & 0 \\
\hline Morganella spp. & 2 & 1 & 0 & 1 & 1 & 1 & 0 & 0 & 2 & 2 & 0 & 0 \\
\hline Myroides spp. & 0 & 0 & 0 & 0 & 1 & 1 & 0 & 0 & 1 & 1 & 0 & 0 \\
\hline Proteus spp. & 6 & 6 & 0 & 0 & 2 & 2 & 0 & 0 & 1 & 0 & 1 & 0 \\
\hline Providencia spp. & 1 & 1 & 0 & 0 & 0 & 0 & 0 & 0 & 0 & 0 & 0 & 0 \\
\hline Psychrobacter spp. & 0 & 0 & 0 & 0 & 0 & 0 & 0 & 0 & 1 & 1 & 0 & 0 \\
\hline Serratia spp. & 1 & 0 & 1 & 0 & 0 & 0 & 0 & 0 & 1 & 0 & 1 & 0 \\
\hline Staphylococcus spp. & 1 & 1 & 0 & 0 & 0 & 0 & 0 & 0 & 0 & 0 & 0 & 0 \\
\hline Stenotrophomonas spp. & 1 & 1 & 1 & 0 & 0 & 0 & 0 & 0 & 0 & 0 & 0 & 0 \\
\hline Trabulsiella spp. & 1 & 0 & 1 & 0 & 0 & 0 & 0 & 0 & 0 & 0 & 0 & 0 \\
\hline Uncultured bacteria clone & 2 & 2 & 0 & 0 & 1 & 1 & 0 & 0 & 0 & 0 & 0 & 0 \\
\hline Total & 32 & 26 & 9 & 2 & 12 & 9 & 3 & 0 & 18 & 14 & 7 & 0 \\
\hline
\end{tabular}

transposons and integrons [31]. Some of these species may be pathogenic strains that have the potential to cause lifethreatening diseases and widespread outbreak. For instance, Zhang et al. [32] have reported that $b l a_{\mathrm{CTX}-\mathrm{M}}$ and $b l a_{\mathrm{TEM}}$ genes in opportunistically pathogenic Klebsiella spp. have been associated with nosocomial infections and outbreak of diarrhea. Therefore, occurrence of these bacteria especially in the drinking water poses a lot of danger to health, economy, and social well-being of consumers. This populace could also be exposed to these genes carrying pathogenic species in food and food products by the use of the contaminated water for domestic purposes, farming, and agriculture. It should also be noted that the fact that these species are multidrug resistance deepens the gravity of the situation. However, from our observation during sampling, the possible source of these MDR bacteria especially in the raw water could be from runoff from agricultural farmlands located very close to some of these constructed dams [4]. Some of these farmlands make use of organic fertilizers which may consist of unmetabolized antibiotics which may eventually get to the water through run-off, which may cause selective pressure on the bacteria in the aquatic systems.

From the bacteria found in Nigeria, many studies have described the occurrence of bla genes among clinical isolates. For example, Akujobi et al. [33] reported bla $a_{\mathrm{TEM}}$ in E. coli while Akinniyi et al. [34] reported the gene not only in E. coli but also among Klebsiella, Salmonella, Citrobacter, Enterobacter, Pseudomonas, and Proteus. The highest prevalence (5.6\%) was also in Klebsiella which is similar to our findings. However, from environmental isolates few studies seem to have been conducted. Moreover, Adelowo et al. [35] reported $b l a_{\mathrm{TEM}}$ in E. coli from well water while Chikwendu et al. [36] described not only $b l a_{\mathrm{TEM}}$ among Pseudomonas from river and aquaculture samples but also $b l a_{\mathrm{SHV}}$. Moreover, this study seems to be the first report describing these genes among a wide diversity of environmental bacteria from Nigeria drinking water distribution systems. It is therefore important to raise public and health worker awareness in terms of prevention of outbreak of MDR infectious pathogens among consumers. This also undermines the need for government agencies controlling these dams and health organizations to initiate measures to effectively control the release of contaminant into the environment. It would also be good to continually isolate bacteria from other water distribution systems in Nigeria and to carry out further molecular testing for the presence of other bla genes, characterize, and determine whether these genes are present on transferable plasmids, transposons, or integrons, which would enhance easy spreading. 


\section{Conclusion}

The occurrence of beta-lactamase producing bacteria and genes in all sampled water in this study, especially treated drinking water, showed that these water distribution systems could serve as a vehicle for transmission of these antibiotic resistance bacteria and genes to consumers, hence, of a great public health concern.

\section{Competing Interests}

The authors declare that they have no competing interests.

\section{Authors' Contributions}

Ayodele T. Adesoji and Adeniyi A. Ogunjobi planned this study. Ayodele T. Adesoji performed the experiment under the guidance of Adeniyi A. Ogunjobi. Ayodele T. Adesoji wrote the paper. All authors read and approved the final paper.

\section{Acknowledgments}

The authors acknowledge Dr. Call Douglas of Paul. G. Allen School of Global Animal Health, Washington State University, USA, who allowed the molecular characterization of the bacteria isolates to be carried out in his laboratory, and Lisa Lorfe who supplied the technical knowhow.

\section{References}

[1] N. J. Ashbolt, "Microbial contamination of drinking water and disease outcomes in developing regions," Toxicology, vol. 198, no. 1-3, pp. 229-238, 2004.

[2] Y. Zhang, C. F. Marrs, C. Simon, and C. Xi, "Wastewater treatment contributes to selective increase of antibiotic resistance among Acinetobacter spp," Science of the Total Environment, vol. 407, no. 12, pp. 3702-3706, 2009.

[3] A. T. Adesoji and A. A. Ogunjobi, "Occurrence of multidrugresistant bacteria in selected water distribution systems in Oyo State, Nigeria," Global Veterinaria, vol. 11, no. 2, pp. 214-224, 2013.

[4] A. A. Timi and O. A. Adeniyi, "Physicochemical properties and occurrence of antibiotic-resistant bacteria in Ife and Ede water distribution systems of southwestern Nigeria," World Applied Sciences Journal, vol. 27, no. 9, pp. 1098-1110, 2013.

[5] A. T. Adesoji, A. A. Ogunjobi, and I. O. Olatoye, "Drinking water distribution systems of dams in Ondo State, Nigeria as reservoir of multi-drug resistant bacteria," World Applied Sciences Journal, vol. 32, pp. 403-414, 2014.

[6] K. Bush, "Characterization of $\beta$-lactamases," Antimicrobial Agents and Chemotherapy, vol. 33, no. 3, pp. 259-263, 1989.

[7] G. S. Singh, " $\beta$-lactam in the new millennium. Part-I: monobactams and carbapenems," Mini-Reviews in Medicinal Chemistry, vol. 4, no. 1, pp. 69-92, 2004.

[8] K. Kumar, S. C. Gupta, Y. Chander, and A. K. Singh, "Antibiotic use in agriculture and its impact on the terrestrial environment," Advances in Agronomy, vol. 87, pp. 1-54, 2005.

[9] P. A. Bradford, "Extended-spectrum $\beta$-lactamases in the 21st century: characterization, epidemiology, and detection of this important resistance threat," Clinical Microbiology Reviews, vol. 14, no. 4, pp. 933-951, 2001.

[10] L. Brusetti, T. Glad, S. Borin et al., "Low prevalence of bla $a_{\mathrm{TEM}}$ genes in Arctic environments and agricultural soil and rhizosphere," Microbial Ecology in Health and Disease, vol. 20, no. 1, pp. 27-36, 2008.

[11] A. T. Adesoji, A. A. Ogunjobi, I. O. Olatoye, and D. R. Douglas, "Prevalence of tetracycline resistance genes among multi-drug resistant bacteria from selected water distribution systems in southwestern Nigeria," Annals of Clinical Microbiology and Antimicrobials, vol. 14, article 35, 2015.

[12] I. S. Henriques, F. Fonseca, A. Alves, M. J. Saavedra, and A. Correia, "Occurrence and diversity of integrons and $\beta$ lactamase genes among ampicillin-resistant isolates from estuarine waters," Research in Microbiology, vol. 157, no. 10, pp. 938947, 2006.

[13] C. Xi, Y. Zhang, C. F. Marrs et al., "Prevalence of antibiotic resistance in drinking water treatment and distribution systems," Applied and Environmental Microbiology, vol. 75, no. 17, pp. 5714-5718, 2009.

[14] U. Govinden, C. Mocktar, P. Moodley, A. W. Sturm, and S. Y. Essack, "Geographical evolution of the CTX-M $\beta$-lactamase: an update," African Journal of Biotechnology, vol. 6, no. 7, pp. 831839, 2007.

[15] C. Dallenne, A. da Costa, D. Decré, C. Favier, and G. Arlet, "Development of a set of multiplex PCR assays for the detection of genes encoding important $\beta$-lactamases in Enterobacteriaceae," Journal of Antimicrobial Chemotherapy, vol. 65, no. 3, pp. 490-495, 2010.

[16] S.-Y. Lu, Y.-L. Zhang, S.-N. Geng et al., "High diversity of extended-spectrum beta-lactamase-producing bacteria in an urban river sediment habitat," Applied and Environmental Microbiology, vol. 76, no. 17, pp. 5972-5976, 2010.

[17] D. Ojdana, P. Sacha, P. Wieczorek et al., "The occurrence of $b l a_{\mathrm{CTX}-\mathrm{M}}, b l a_{\mathrm{SHV}}$, and $b l a_{\mathrm{TEM}}$ genes in extended-spectrum $\beta$ lactamase-positive strains of Klebsiella pneumoniae, Escherichia coli, and Proteus mirabilis in Poland," International Journal of Antibiotics, vol. 2014, Article ID 935842, 7 pages, 2014.

[18] A. T. Adesoji, A. A. Ogunjobi, and I. O. Olatoye, "Molecular characterization of selected multidrug resistant Pseudomonas from water distribution systems in southwestern Nigeria," Annals of Clinical Microbiology and Antimicrobials, vol. 14, article 39, 2015.

[19] F. Luzzaro, M. Mezzatesta, C. Mugnaioli et al., "Trends in production of extended-spectrum $\beta$-lactamases among enterobacteria of medical interest: report of the second Italian nationwide survey," Journal of Clinical Microbiology, vol. 44, no. 5, pp. 1659-1664, 2006.

[20] T. Spanu, F. Luzzaro, M. Perilli et al., "Occurrence of extendedspectrum $\beta$-lactamases in members of the family Enterobacteriaceae in Italy: implications for resistance to $\beta$-lactams and other antimicrobial drugs," Antimicrobial Agents and Chemotherapy, vol. 46, no. 1, pp. 196-202, 2002.

[21] S. Tissera and S. M. Lee, "Isolation of extended spectrum $\beta$ lactamase (ESBL) producing bacteria from urban surface waters in Malaysia," Malaysian Journal of Medical Sciences, vol. 20, no. 3, pp. 14-22, 2013.

[22] K. Kadlec, I. Wiegand, C. Kehrenberg, and S. Schwarz, "Studies on the mechanisms of $\beta$-lactam resistance in Bordetella bronchiseptica," Journal of Antimicrobial Chemotherapy, vol. 59, no. 3, pp. 396-402, 2007. 
[23] S. Mahrouki, H. Chihi, A. Bourouis et al., "Nosocomial dissemination of plasmids carrying $b l a_{\mathrm{TEM}-24}, b l a_{\mathrm{DHA}-1}, a a c\left(6^{\prime}\right)-I b-c r$, and qnrA6 in Providencia spp. strains isolated from a Tunisian hospital," Diagnostic Microbiology and Infectious Disease, vol. 81, no. 1, pp. 50-52, 2015.

[24] M. B. Avison, C. J. von Heldreich, C. S. Higgins, P. M. Bennett, and T. R. Walsh, "A TEM-2 $\beta$-lactamase encoded on an active Tnl-like transposon in the genome of a clinical isolate of Stenotrophomonas maltophilia," Journal of Antimicrobial Chemotherapy, vol. 46, no. 6, pp. 879-884, 2000.

[25] L. C. Balsalobre, M. Dropa, D. E. de Oliveira et al., "Presence of bla $a_{\mathrm{TEM}-116}$ gene in environmental isolates of Aeromonas hydrophila and Aeromonas jandaei from Brazil," Brazilian Journal of Microbiology, vol. 41, no. 3, pp. 718-719, 2010.

[26] Z. Yang, W. Liu, Q. Cui et al., "Prevalence and detection of Stenotrophomonas maltophilia carrying metallo- $\beta$-lactamase blaL1 in Beijing, China," Frontiers in Microbiology, vol. 5, article 692, 2014

[27] A. Karim, L. Poirel, S. Nagarajan, and P. Nordmann, "Plasmidmediated extended-spectrum $\beta$-lactamase (CTX-M-3 like) from India and gene association with insertion sequence ISEcpl," FEMS Microbiology Letters, vol. 201, no. 2, pp. 237-241, 2001.

[28] C. J. Munday, G. M. Whitehead, N. J. Todd, M. Campbell, and P. M. Hawkey, "Predominance and genetic diversity of community- and hospital-acquired CTX-M extendedspectrum $\beta$-lactamases in York, UK," Journal of Antimicrobial Chemotherapy, vol. 54, no. 3, pp. 628-633, 2004.

[29] M. Shahid, A. Singh, F. Sobia et al., " $b l a_{\mathrm{CTX}-\mathrm{M}}, b l a_{\mathrm{TEM}}$, and $b l a_{\mathrm{SHV}}$ in Enterobacteriaceae from North-Indian tertiary hospital: high occurrence of combination genes," Asian Pacific Journal of Tropical Medicine, vol. 4, no. 2, pp. 101-105, 2011.

[30] J. K. Bailey, J. L. Pinyon, S. Anantham, and R. M. Hall, "Distribution of the bla $a_{\mathrm{TEM}}$ gene and $b l a_{\mathrm{TEM}}$-containing transposons in commensal Escherichia coli," Journal of Antimicrobial Chemotherapy, vol. 66, no. 4, pp. 745-751, 2011.

[31] M. L. Güerri, A. Aladueña, A. Echeíta, and R. Rotger, "Detection of integrons and antibiotic-resistance genes in Salmonella enterica serovar Typhimurium isolates with resistance to ampicillin and variable susceptibility to amoxicillin-clavulanate," International Journal of Antimicrobial Agents, vol. 24, no. 4, pp. 327-333, 2004.

[32] Y. Zhang, H. Zhou, X.-Q. Shen, P. Shen, Y.-S. Yu, and L.-J. Li, "Plasmid-borne armA methylase gene, together with $b l a_{\mathrm{CTX}-\mathrm{M}-15}$ and bla $_{\mathrm{TEM}-1}$, in a Klebsiella oxytoca isolate from China," Journal of Medical Microbiology, vol. 57, no. 10, pp. 1273-1276, 2008.

[33] C. N. Akujobi, C. C. Ezeanya, and N. I. Aghanya, "Detection of cefotaximase genes of beta lactamase among clinical isolates of Escherichia coli in a university teaching hospital, Nigeria," Journal of Medical Sciences, vol. 12, no. 7, pp. 244-247, 2012.

[34] A. P. Akinniyi, O. Afolabi, B. A. Iwalokun, E. Oluwaseun, and K. O. Onagbesan, "Clonal dissemination of $b l a_{\text {TEM }} \beta$-lactamase strains among enteric isolates in Abeokuta, Nigeria," Research Journal of Microbiology, vol. 6, no. 12, pp. 919-925, 2011.

[35] O. O. Adelowo, O. E. Fagade, and Y. Agersø, "Antibiotic resistance and resistance genes in Escherichia coli from poultry farms, southwest Nigeria," Journal of Infection in Developing Countries, vol. 8, no. 9, pp. 1103-1112, 2014.

[36] C. I. Chikwendu, S. N. Ibe, and G. C. Okpokwasili, "Detection of $b l a_{\mathrm{SHV}}$ and $b l a_{\mathrm{TEM}}$ beta-lactamase genes in multi-resistant Pseudomonas isolates from environmental sources," African Journal of Microbiology Research, vol. 5, no. 15, pp. 2067-2074, 2011. 

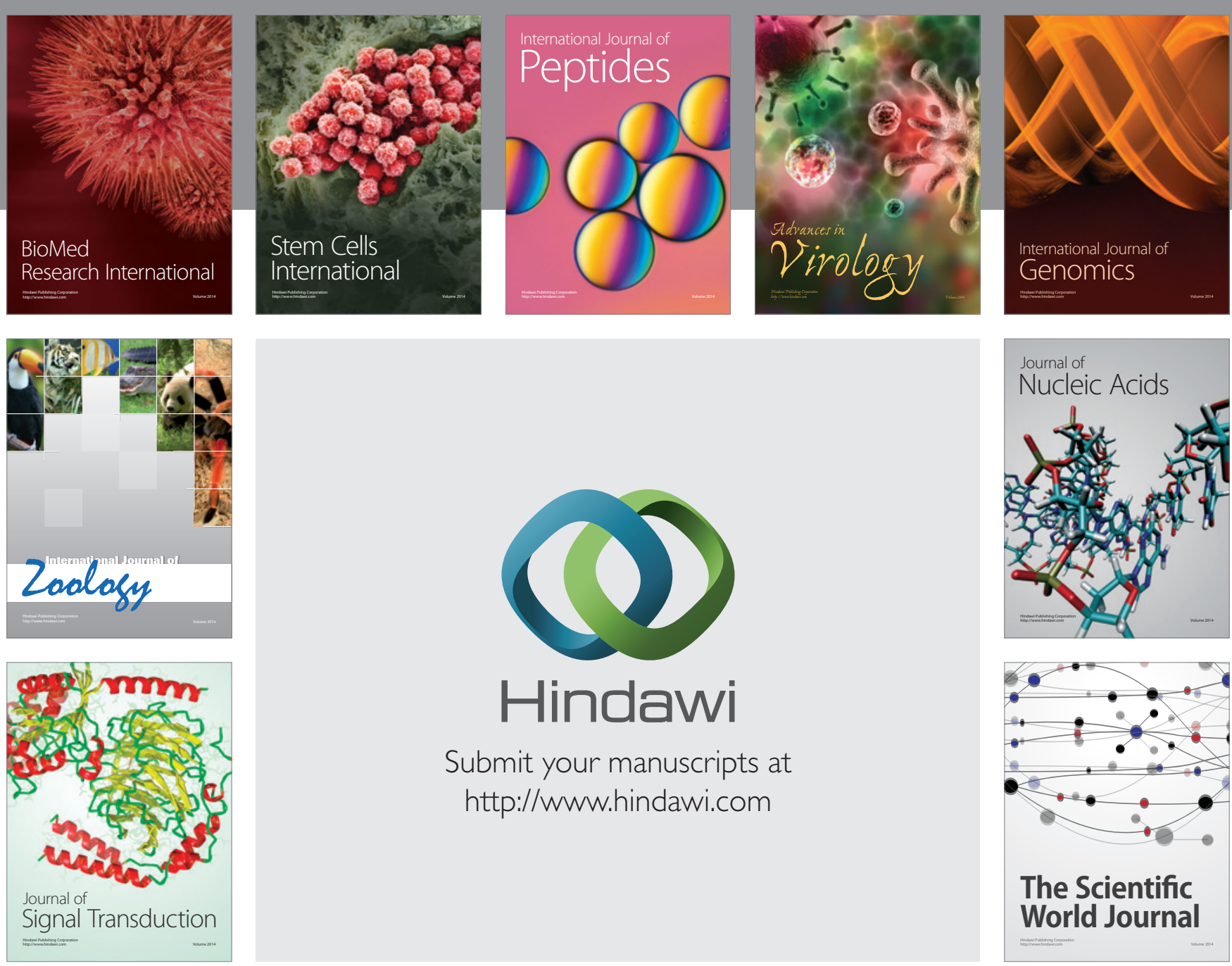

Submit your manuscripts at

http://www.hindawi.com
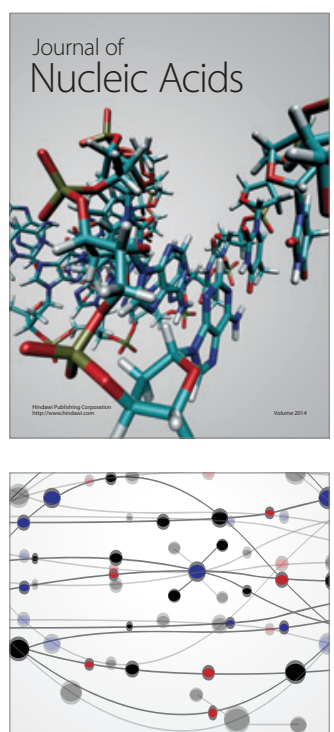

The Scientific World Journal
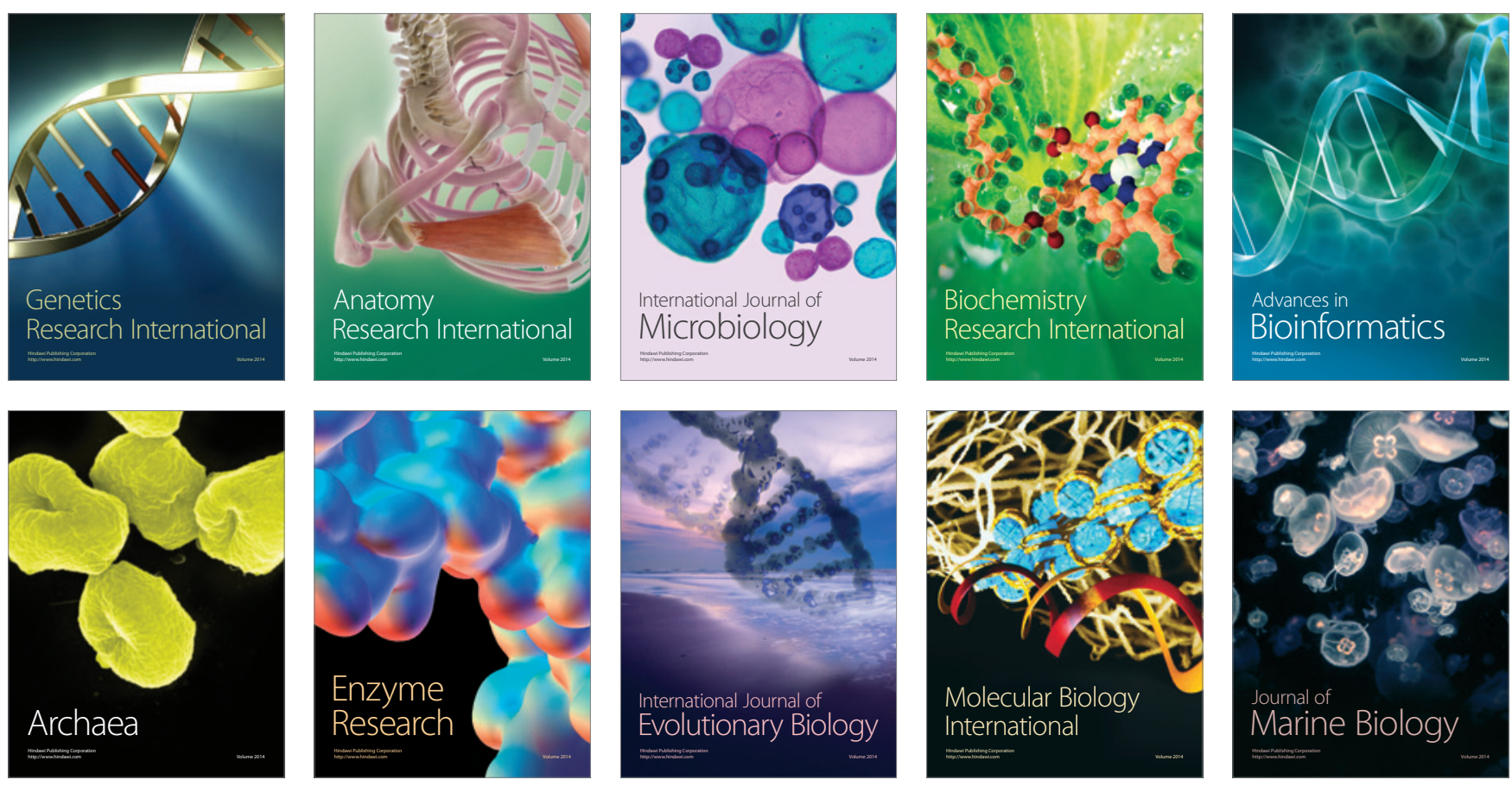